Research Article

\title{
Ultrasensitive Anomalous Hall Effect in Ta/CoFe/Oxide/Ta Multilayers
}

\author{
Guang Yang, Yongye Li, Xi Chen, Jingyan Zhang, and Guanghua Yu \\ Department of Materials Physics and Chemistry, University of Science and Technology Beijing, Beijing 100083, China \\ Correspondence should be addressed to Guanghua Yu; ghyu@mater.ustb.edu.cn
}

Received 6 October 2016; Accepted 22 November 2016

Academic Editor: Hao Zeng

Copyright (C) 2016 Guang Yang et al. This is an open access article distributed under the Creative Commons Attribution License, which permits unrestricted use, distribution, and reproduction in any medium, provided the original work is properly cited.

\begin{abstract}
Ultrahigh anomalous Hall sensitivity has been demonstrated in $\mathrm{Ta} / \mathrm{CoFe} / O x i d e / \mathrm{Ta}$ multilayers. By changing oxides ( $\mathrm{MgO}$ and $\mathrm{HfO}_{2}$ ) and annealing temperature, different annealing dependence of sensitivity was found in $\mathrm{MgO}$-sample and $\mathrm{HfO}_{2}$-sample. For the $\mathrm{MgO}$-sample, the anomalous Hall sensitivity reaches $18792 \Omega / \mathrm{T}$ in the as-deposited state and significantly reduces as annealing temperature increases. On the contrary, the sensitivity of the as-deposited $\mathrm{HfO}_{2}$-sample is only $765 \Omega / \mathrm{T}$, while it remarkably increases with annealing temperature increasing, finally reaching $14741 \Omega / \mathrm{T}$ at $240^{\circ} \mathrm{C}$. The opposite variation of anomalous sensitivity in two samples originates from the different change of magnetic anisotropy and anomalous Hall resistance during the annealing process. Our study provides a new perspective that both the choice of oxide material and the optimization of annealing treatment are important to the anomalous Hall sensitivity.
\end{abstract}

\section{Introduction}

Magnetic sensors are playing an increasing important role in daily life and industrial production, with their wide applications ranging from read heads in the hard disk [1], to the speed and rotation angle detectors in the automotive industry [2], and even to the detection of DNA and proteins [3]. The current design of magnetic sensors is based on the Hall effect in semiconductor materials or magnetoresistive effect including anisotropy magnetoresistance (AMR), giant magnetoresistance (GMR), and tunneling magnetoresistance (TMR) in magnetic materials. However, the sensors based on Hall effect and AMR effect always suffer a lower sensitivity. On the other hand, although high sensitivity can be obtained in GMR- and TMR-based sensors, the complex fabrication process with higher costs is also an obstacle. Recently, the anomalous Hall effect (AHE) of ferromagnets has attracted enormous attention owning to the abundant physics $[4,5]$ and potential applications [6, 7]. In 2007, Zhu and Cai [8] first demonstrated an anomalous Hall sensitivity as high as $1200 \Omega / \mathrm{T}$ in $[\mathrm{CoFe} / \mathrm{Pt}]_{n}$ multilayers, which is better than the conventional semiconductor Hall sensitivity (about $1000 \Omega / T$ ). Subsequently, the strategy adapted to achieve a higher sensitivity was by using ultrathin ferromagnetic films/multilayers with enhanced spin-orbit scattering and tailored magnetic anisotropy that enables large anomalous Hall resistance and low saturation field [9-13]. In particular, Lu et al. [11] obtained a sensitivity of $12000 \Omega / \mathrm{T}$ in $\mathrm{SiO}_{2} / \mathrm{FePt} / \mathrm{SiO}_{2}$ sandwich structure films with optimized FePt composition and thickness. Zhu et al. [12] demonstrated a sensitivity of $23760 \Omega / \mathrm{T}$ in $\mathrm{MgO} / \mathrm{CoFeB} / \mathrm{Ta} / \mathrm{MgO}$ multilayers by tuning the thickness of $\mathrm{CoFeB}$ and adjacent Ta layer. More excitingly, a very recent study has reported the anomalous Hall sensitivity up to $10^{6} \Omega / \mathrm{T}$, which is two orders higher than the best of semiconductors [13].

Although the achieved ultrahigh sensitivity is remarkable, the compatibility between AHE materials and CMOS technology still needs further consideration. For example, heavy metals such as Pt are always used in AHE materials to enhance the spin-orbit scattering for a large anomalous Hall resistance, while it will cause a terrible shunting effect as well as increased costs. The $\mathrm{CoFeB} / \mathrm{MgO}$ heterostructure seems a more promising material system, while the commonly used oxides in CMOS technology are high- $k$ materials such as $\mathrm{SiO}_{2}$ and $\mathrm{HfO}_{2}$. From the application point of view, it is better to introduce the same high- $k$ oxides into the AHE materials. Last but not least, AHE materials generally need 
additional annealing to exhibit a high sensitivity. Considering the postannealing is also essential to CMOS technology, it is necessary to further optimize the annealing process.

In this work, we demonstrate the ultrasensitive AHE in $\mathrm{Ta} / \mathrm{CoFe} / \mathrm{Oxide} / \mathrm{Ta}$ multilayers. By changing oxides ( $\mathrm{MgO}$ and $\mathrm{HfO}_{2}$ ) and annealing temperature $\left(T_{a}\right)$, opposite $T_{a}$ dependence of sensitivity was found in $\mathrm{MgO}$-sample and $\mathrm{HfO}_{2}$-sample. For the MgO-sample, the anomalous Hall sensitivity reaches $18792 \Omega / \mathrm{T}$ in the as-deposited state and significantly reduces as $T_{a}$ increases. On the contrary, the sensitivity of the as-deposited $\mathrm{HfO}_{2}$-sample is only $765 \Omega / \mathrm{T}$, while it remarkably increases with $T_{a}$ increasing, finally reaching $14741 \Omega / \mathrm{T}$ at $240^{\circ} \mathrm{C}$. Based on the angular dependent ferromagnetic resonance (FMR) measurements and temperature dependent transport measurements, the different change of sensitivity in two samples comes from the different temperature dependence of the anomalous Hall resistance and the magnetic anisotropy. This study gives new insights that the choice of oxides and the optimization of $T_{a}$ are both important to obtain an ultrahigh anomalous Hall sensitivity.

\section{Experiments}

All samples were deposited on Si substrates by magnetron sputtering at room temperature. The sample structure is $\mathrm{Ta}(0.8) / \mathrm{Co}_{20} \mathrm{Fe}_{80}(0.8) /$ Oxide(0.8)/Ta(1.0) (all in $\mathrm{nm}$ ), where the oxide is $\mathrm{MgO}$ or $\mathrm{HfO}_{2}$. Thermal annealing was carried out in a vacuum furnace (better than $3 \times 10^{-7}$ Torr) for $15 \mathrm{~min}$ without external magnetic fields. Hall bars were patterned by optical lithography combined with $\mathrm{Ar}^{+}$milling for transport measurements in a physical property measurement system. FMR measurements were performed in an electron spin resonance spectrometer (JEOL ESR FA-200) at X-band (9.0 GHz).

\section{Results and Discussions}

The anomalous Hall sensitivity is defined as $S=d R_{x y} / d H \approx$ $R_{\mathrm{AH}} / H_{s}[12,14]$, where $H_{s}$ is the perpendicular saturation field and $R_{\mathrm{AH}}$ is the saturated anomalous Hall resistance that can be obtained via a linear extrapolation of $R_{x y}$ at high field to zero field. The inset of Figure 1 exhibits the anomalous Hall loops of sample $\mathrm{Ta}(0.8) / \mathrm{Co}_{20} \mathrm{Fe}_{80}(0.8) / \mathrm{MgO}(0.8) / \mathrm{Ta}(1.0)$ (in $\mathrm{nm})$ in the as-deposited and different annealed states, from which the corresponding value of $S$ is calculated. As a result, Figure 1 shows the sensitivity $S$ as a function of the annealing temperature $T_{a}$. When $T_{a}$ is $25^{\circ} \mathrm{C}$ (as-deposited state), $S$ of $\mathrm{MgO}$-sample has reached $18792 \Omega / \mathrm{T}$. Nevertheless, the value of $S$ decreases significantly with the increase of $T_{a}$. When $T_{a}$ reaches $140^{\circ} \mathrm{C}$, the value of $S$ is $8145 \Omega / \mathrm{T}$, decreasing $57 \%$ with respect to that in the as-deposited state. As $T_{a}$ further increases to $240^{\circ} \mathrm{C}$, the value of $S$ is only $2572 \Omega / \mathrm{T}$.

In contrast, Figure 2 shows $S$ as a function of $T_{a}$ for sample $\mathrm{Ta}(0.8) / \mathrm{Co}_{20} \mathrm{Fe}_{80}(0.8) / \mathrm{HfO}_{2}(0.8) / \mathrm{Ta}(1.0)$ (in nm). Different from the MgO-sample, the value of $S$ in the as-deposited $\mathrm{HfO}_{2}$ sample is only $765 \Omega / \mathrm{T}$. When $T_{a}$ increases to $180^{\circ} \mathrm{C}$, the value of $S$ appears almost unchanged. However, as $T_{a}$ is above $200^{\circ} \mathrm{C}$, the value of $S$ increases dramatically. When $T_{a}$ reaches $240^{\circ} \mathrm{C}$, the value of $S$ is $14741 \Omega / \mathrm{T}$, which is about 19

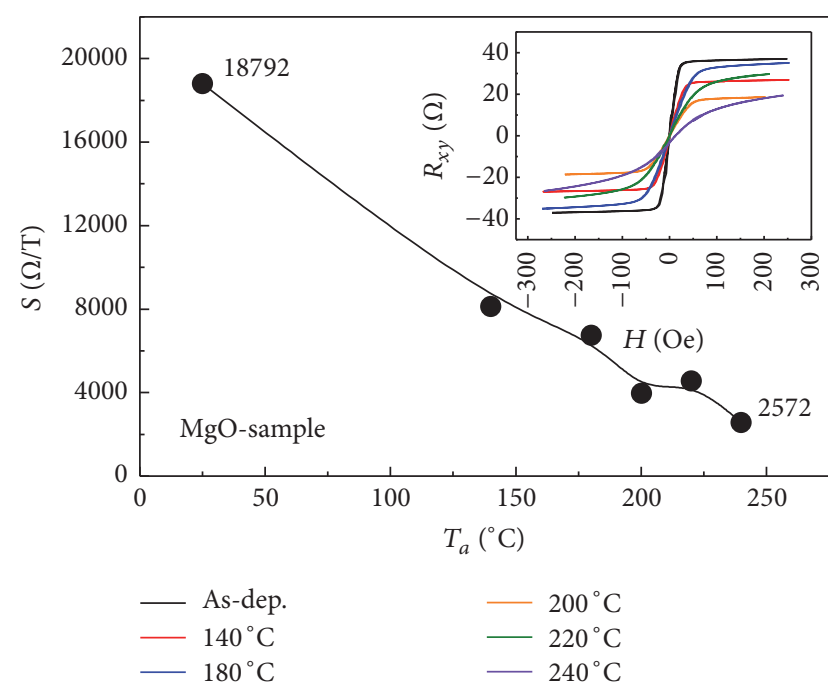

FIGURE 1: Annealing temperature dependence of the anomalous Hall sensitivity for the sample $\mathrm{Ta}(0.8) / \mathrm{Co}_{20} \mathrm{Fe}_{80}(0.8) / \mathrm{MgO}(0.8) / \mathrm{Ta}(1.0)$ (in $\mathrm{nm}$ ). Inset: anomalous Hall loops of the sample in the asdeposited and different annealed states.

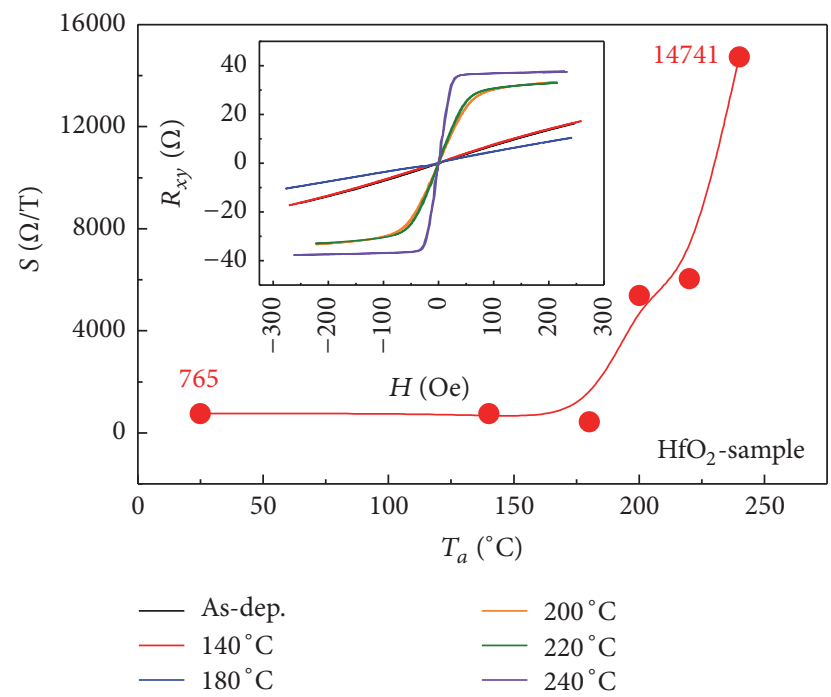

FIGURE 2: Annealing temperature dependence of the anomalous Hall sensitivity for the sample $\mathrm{Ta}(0.8) / \mathrm{Co}_{20} \mathrm{Fe}_{80}(0.8) / \mathrm{HfO}_{2}(0.8) /$ $\mathrm{Ta}(1.0)$ (in $\mathrm{nm}$ ). Inset: anomalous Hall loops of the sample in the as-deposited and different annealed states.

times larger than that in the as-deposited state. It is interesting to find that the variation trend of $S$ with respect to $T_{a}$ is opposite in the MgO-sample and $\mathrm{HfO}_{2}$-sample. To further illustrate the difference, four typical samples were chosen as below: as-deposited $\mathrm{MgO}$-sample, $240^{\circ} \mathrm{C}$ annealed $\mathrm{MgO}$ sample, as-deposited $\mathrm{HfO}_{2}$-sample, and $240^{\circ} \mathrm{C}$ annealed $\mathrm{HfO}_{2}$-sample.

As shown in Figure 3, the detailed $R_{x y}-H$ curves of the above four samples are presented. In Figure 3(a), the curve of the as-deposited $\mathrm{MgO}$-sample (black one) shows an obvious linear response without magnetic hysteresis. The saturated 


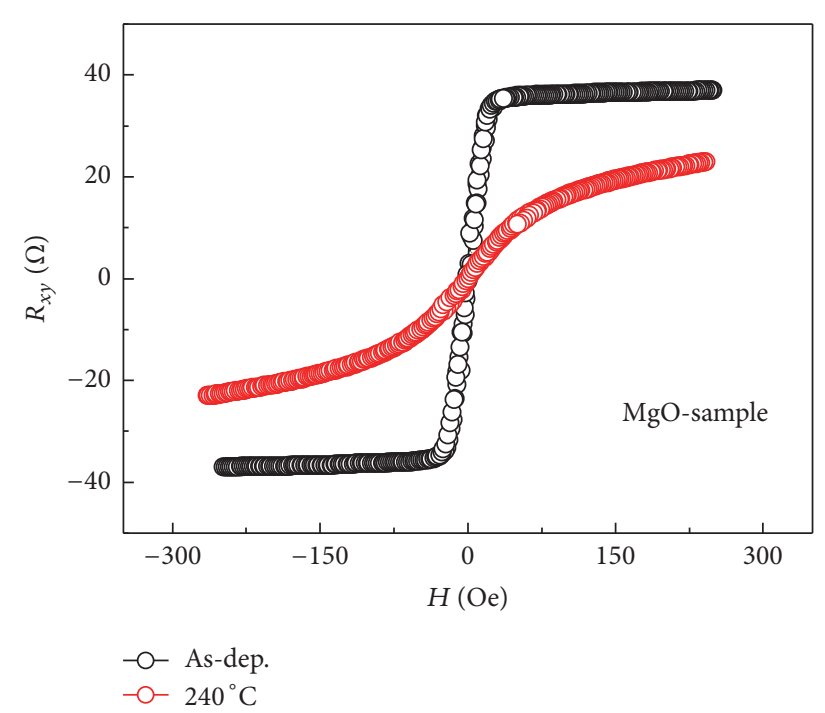

(a)

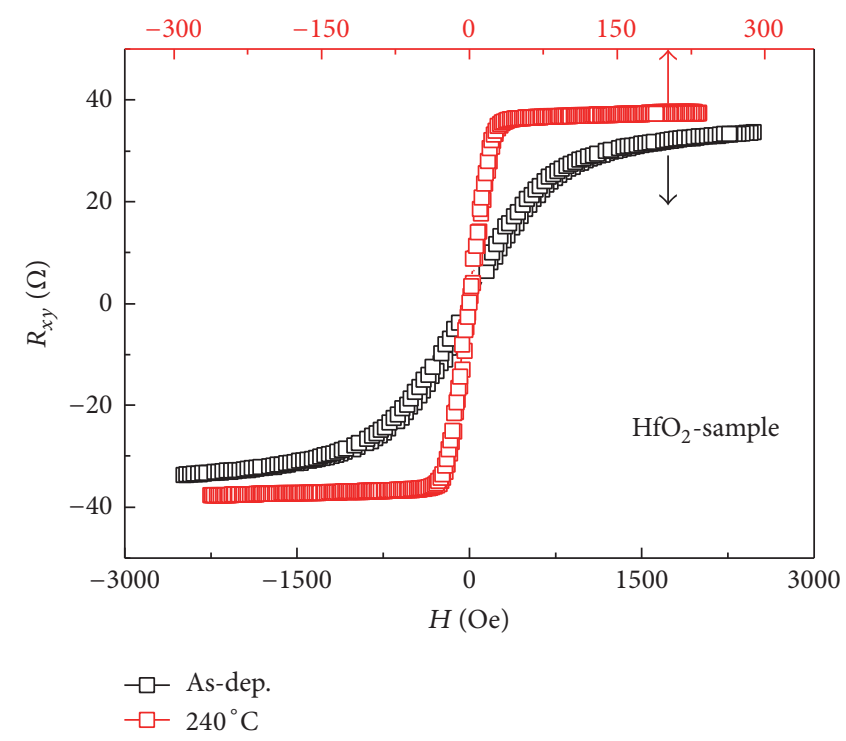

(b)

Figure 3: (a) $R_{x y}-H$ curves for sample $\mathrm{Ta}(0.8) / \mathrm{Co}_{20} \mathrm{Fe}_{80}(0.8) / \mathrm{MgO}(0.8) / \mathrm{Ta}(1.0)$ (in $\mathrm{nm}$ ) in the as-deposited and $240^{\circ} \mathrm{C}$ annealed states. (b) $R_{x y}-H$ curves for sample $\mathrm{Ta}(0.8) / \mathrm{Co}_{20} \mathrm{Fe}_{80}(0.8) / \mathrm{HfO}_{2}(0.8) / \mathrm{Ta}(1.0)$ (in $\mathrm{nm}$ ) in the as-deposited and $240^{\circ} \mathrm{C}$ annealed states.

anomalous Hall resistance $R_{\mathrm{AH}}$ is $35.8 \Omega$ and the perpendicular saturation field $H_{s}$ is $20 \mathrm{Oe}$. By annealing at $240^{\circ} \mathrm{C}$, the linear shape of the curve began to degrade, with $R_{\mathrm{AH}}$ decreasing to $14.2 \Omega$ and $H_{s}$ increasing to 150 Oe. Both the reduced $R_{\mathrm{AH}}$ and the increased $H_{s}$ are detrimental to the sensitivity, leading to a significant decrease of $S$ from $18792 \Omega /$ T to $2572 \Omega /$ T. Figure $3(\mathrm{~b})$ shows the $R_{x y}-H$ curves of the as-deposited and $240^{\circ} \mathrm{C}$ annealed $\mathrm{HfO}_{2}$-samples. The values of $R_{\mathrm{AH}}$ and $H_{s}$ for the as-deposited sample are $28.5 \Omega$ and $1000 \mathrm{Oe}$. By annealing at $240^{\circ} \mathrm{C}$, the value of $R_{\mathrm{AH}}$ reaches $36.4 \Omega$ while the value of $H_{s}$ decreases to $30 \mathrm{Oe}$. Both the increased $R_{\mathrm{AH}}$ and the reduced $H_{s}$ are beneficial to an ultrahigh sensitivity, leading to a significant increase of $S$ from $765 \Omega / \mathrm{T}$ to $14741 \Omega / \mathrm{T}$.
It is well known that the perpendicular saturation field is related to the magnetic anisotropy of the films. During the annealing process, the volume anisotropy as well as the interfacial anisotropy is likely to change $[15,16]$. In order to characterize the evolution of magnetic anisotropy in the $\mathrm{MgO}$ - and $\mathrm{HfO}_{2}$-samples, out-of-plane angular dependent FMR measurements were performed. The typical FMR differential absorption spectrum is shown in the inset of Figure 4(a), where the resonance field $H_{\text {res }}$ and peak-to-peak linewidth $\Delta H_{\mathrm{pp}}$ are defined. Figure $4(\mathrm{a})$ presents the outof-plane angular dependent $H_{\text {res }}$ for the as-deposited MgOsample. Here, the angle $\theta_{H}$ is defined as the direction of applied magnetic field with respect to the film normal. The value of $H_{\text {res }}$ can be fitted by Kittel's formula:

$$
\begin{aligned}
f & =\frac{\gamma}{2 \pi} \sqrt{f_{1} f_{2}} \\
& =\frac{\gamma}{2 \pi} \sqrt{H_{\mathrm{res}} \cos \left(\theta_{H}-\theta\right)+H_{1} \cos ^{2} \theta-H_{2} \cos ^{4} \theta} \sqrt{H_{\mathrm{res}} \cos \left(\theta_{H}-\theta\right)+H_{1} \cos 2 \theta+H_{2}\left(3 \cos ^{2} \theta \sin ^{2} \theta-\cos ^{4} \theta\right)},
\end{aligned}
$$

where $H_{1}=2 K_{1} / M_{s}+4 K_{2} / M_{s}-4 \pi M_{s}$ and $H_{2}=4 K_{2} / M_{s}$. $K_{1}, K_{2}, M_{s}$, and $\theta$ are the first-order, second-order uniaxial anisotropy constant, the saturation magnetization, and the equilibrium angle of the magnetization vector with respect to film normal, respectively. $f=9.0 \mathrm{GHz}$ is the frequency of AC magnetic fields in the machine. $\gamma$ is the gyromagnetic ratio given as $\gamma=g \mu_{B} / \hbar$, where $g, \mu_{B}$, and $\hbar$ are Landé factor, Bohr magneton, and Planck's constant, respectively. As shown in Figure 4(a), the experimental value of $H_{\text {res }}$ as a function of $\theta_{H}$ can be well fitted, where above parameters can be obtained. Consequently, the fitting parameters $g, M_{s}$, $K_{1}, K_{2}$, the effective magnetic anisotropy constant $K_{\text {eff }}=$ $K_{1}-2 \pi M_{s}^{2}$, and the effective anisotropy filed $H_{\text {eff }}=2 K_{\text {eff }} / M_{s}$ calculated from Figures 4(a)-4(d) are listed in Table 1.

From Table 1, it is clearly seen that the variation trend of magnetic anisotropy is different in the $\mathrm{MgO}$-sample and $\mathrm{HfO}_{2}$-sample. For the as-deposited $\mathrm{MgO}$-sample, both values of the effective magnetic anisotropy constant $K_{\text {eff }}$ and the second-order uniaxial anisotropy constant $K_{2}$ are positive, indicating the sample has perpendicular magnetic anisotropy 
TABLE 1: Fitting parameters deduced from (1) in the four samples.

\begin{tabular}{lcccccc}
\hline & $g$ & $M_{s}\left(\times 10^{3} \mathrm{emu} / \mathrm{cm}^{3}\right)$ & $K_{1}\left(\times 10^{6} \mathrm{erg} / \mathrm{cm}^{3}\right)$ & $K_{2}\left(\times 10^{4} \mathrm{erg} / \mathrm{cm}^{3}\right)$ & $K_{\mathrm{eff}}\left(\times 10^{5} \mathrm{erg} / \mathrm{cm}^{3}\right)$ & $H_{\mathrm{eff}}(\mathrm{Oe})$ \\
\hline $\mathrm{MgO}$ as-dep. & 2.01 & 1.13 & 8.02 & 8.89 & 10.15 & -43 \\
$\mathrm{MgO} 240^{\circ} \mathrm{C}$ & 1.99 & 1.14 & 7.76 & 2.47 & -7.09 & -716.92 \\
$\mathrm{HfO}_{2}$ as-dep. & 2.04 & 1.14 & 7.71 & 1.01 & -5.4 & -942.60 \\
$\mathrm{HfO}_{2} 240^{\circ} \mathrm{C}$ & 2.04 & 1.12 & 8.10 & 2.07 & 369.26 \\
\hline
\end{tabular}

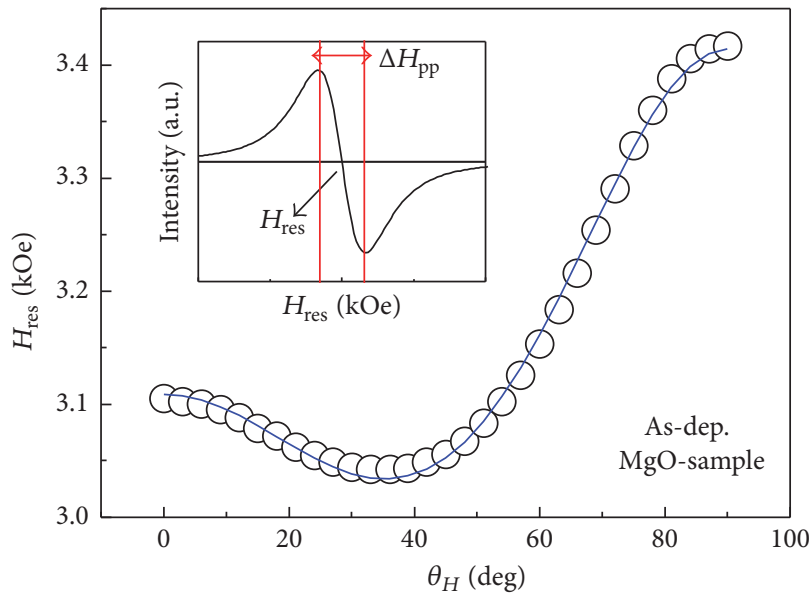

(a)

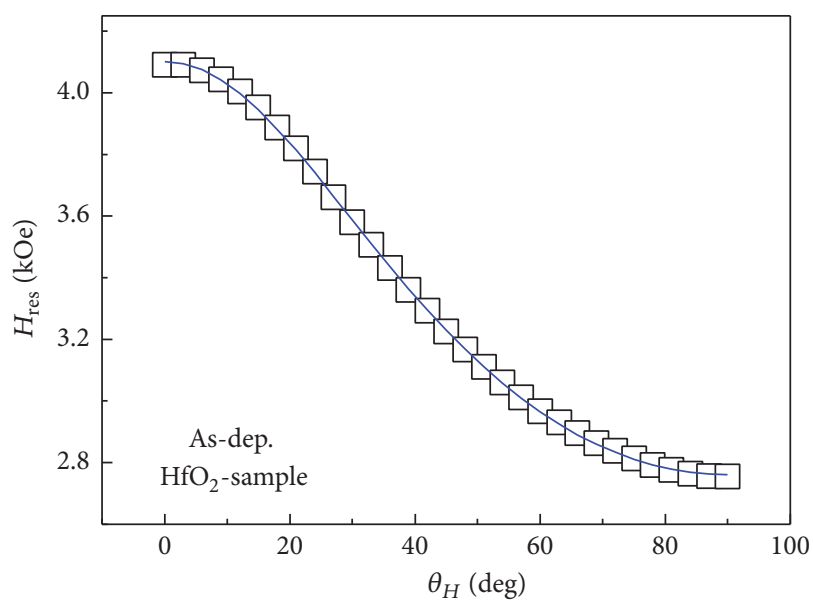

(c)

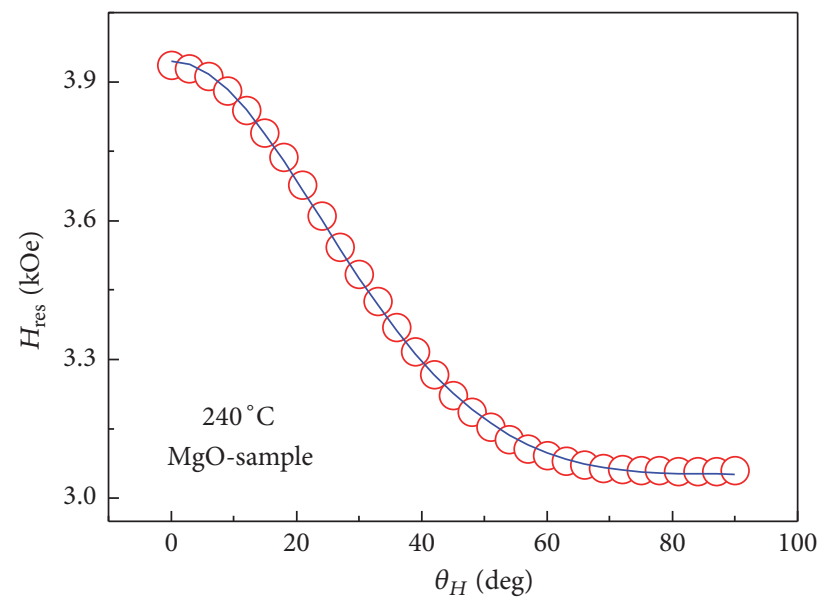

(b)

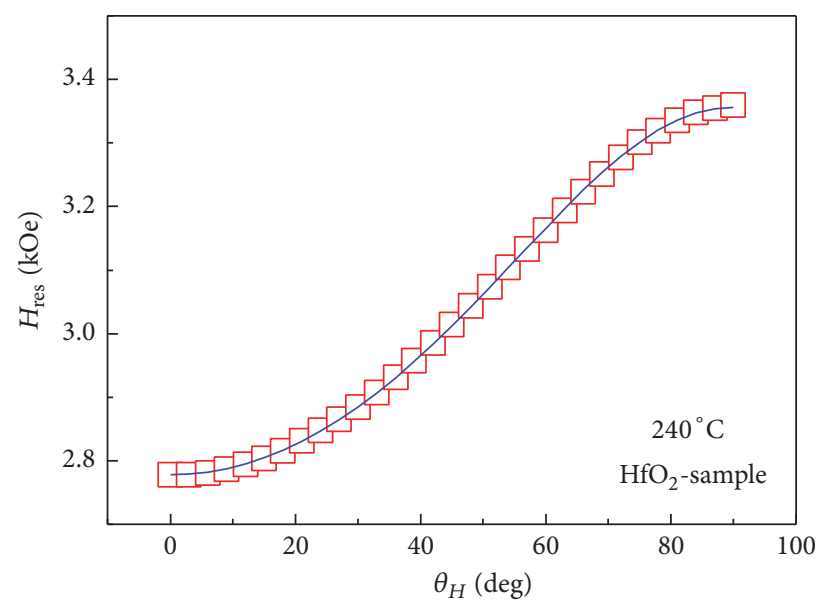

(d)

Figure 4: (a)-(b) Out-of-plane angular dependent resonance fields $H_{\text {res }}$ for sample $\mathrm{Ta}(0.8) / \mathrm{Co}_{20} \mathrm{Fe}_{80}(0.8) / \mathrm{MgO}(0.8) / \mathrm{Ta}(1.0)$ (in $\mathrm{nm}$ ) in the as-deposited and $240^{\circ} \mathrm{C}$ annealed states. Hollow circles and solid lines represent experimental data and theoretical fitting of $H_{\text {res }}$. Inset: typical FMR differential absorption spectra where the resonance field $H_{\text {res }}$ and peal-to-peak linewidth $\Delta H_{\mathrm{pp}}$ are defined. (c)-(d) Out-of-plane angular dependent resonance fields $H_{\text {res }}$ for sample $\mathrm{Ta}(0.8) / \mathrm{Co}_{20} \mathrm{Fe}_{80}(0.8) / \mathrm{HfO}_{2}(0.8) / \mathrm{Ta}(1.0)$ (in nm) in the as-deposited and $240^{\circ} \mathrm{C}$ annealed states. Hollow diamonds and solid lines represent experimental data and theoretical fitting of $H_{\text {res }}$.

(PMA) [17]. For the sample with PMA, the perpendicular direction is the easy magnetization axis; thus the perpendicular saturation filed $H_{s}$ is small. It is also important to point out that since the calculated effective anisotropy field $H_{\text {eff }}$ is very small (only about $94 \mathrm{Oe}$ ), the $R_{x y}-H$ curve will not exhibit the obvious coercivity. For the $240^{\circ} \mathrm{C}$ annealed $\mathrm{MgO}$-sample, the calculated values of $K_{\text {eff }}$ and $K_{2}$ are $-4.09 \times$ $10^{5} \mathrm{erg} / \mathrm{cm}^{3}$ and $1.02 \times 10^{5} \mathrm{erg} / \mathrm{cm}^{3}$, respectively. Considering the value of $K_{\text {eff }}$ is negative and $K_{2}<-(1 / 2) K_{\text {eff }}$, the annealed
$\mathrm{MgO}$-sample has in-plane magnetic anisotropy (IMA) [17]. For the sample with IMA, the perpendicular direction is the difficult magnetization axis; thus the value of $H_{s}$ will be very large. On the other hand, for the as-deposited $\mathrm{HfO}_{2}$-sample, the value of $K_{\text {eff }}$ is negative and $K_{2}<-(1 / 2) K_{\text {eff }}$, representing a typical IMA character. However, by annealing at $240^{\circ} \mathrm{C}$, both the values of $K_{\text {eff }}$ and $K_{2}$ change to positive, indicating the $240^{\circ} \mathrm{C}$ annealed $\mathrm{HfO}_{2}$ sample has PMA with a small $H_{s}$. Therefore, the variation trend of magnetic anisotropy 


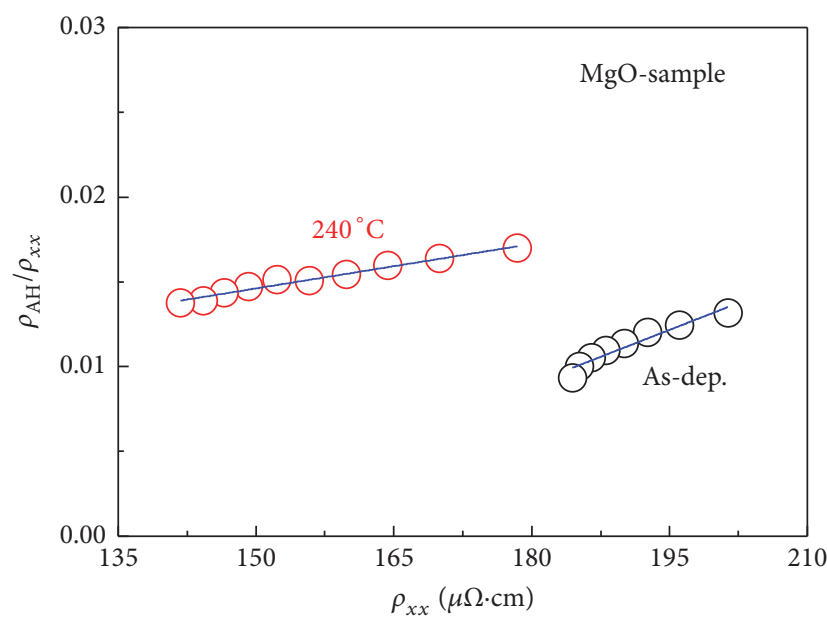

(a)

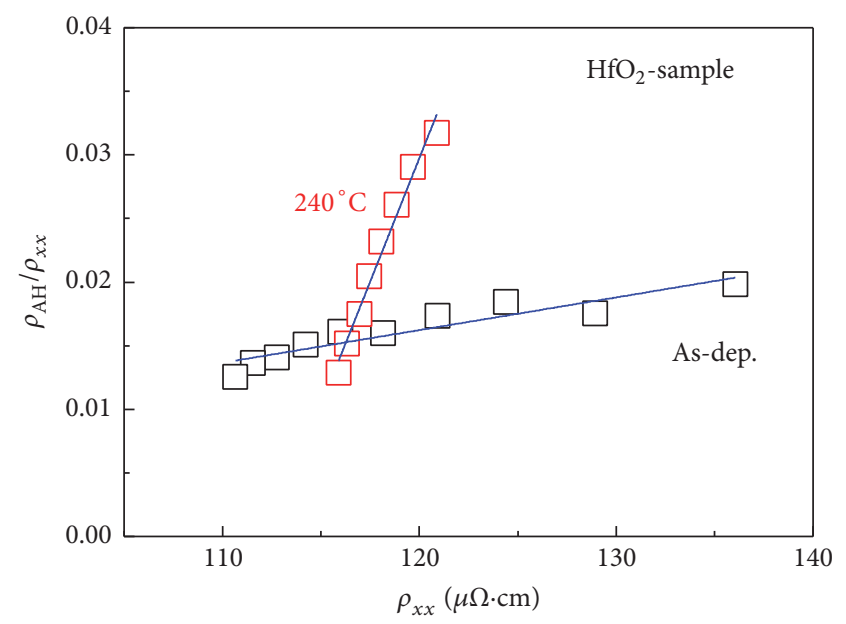

(b)

Figure 5: (a) $\rho_{\mathrm{AH}} / \rho_{x x}$ versus $\rho_{x x}$ for sample $\mathrm{Ta}(0.8) / \mathrm{Co}_{20} \mathrm{Fe}_{80}(0.8) / \mathrm{MgO}(0.8) / \mathrm{Ta}(1.0)$ (in $\mathrm{nm}$ ) in the as-deposited and $240^{\circ} \mathrm{C}$ annealed states. (b) $\rho_{\mathrm{AH}} / \rho_{x x}$ versus $\rho_{x x}$ for sample $\mathrm{Ta}(0.8) / \mathrm{Co}_{20} \mathrm{Fe}_{80}(0.8) / \mathrm{HfO}_{2}(0.8) / \mathrm{Ta}(1.0)$ (in nm) in the as-deposited and $240^{\circ} \mathrm{C}$ annealed states.

during annealing is opposite in the $\mathrm{MgO}$-sample and $\mathrm{HfO}_{2}$ sample. For MgO-sample, the magnetic anisotropy changes from PMA to IMA, resulting in a significant increase of $H_{s}$, while, for $\mathrm{HfO}_{2}$-sample, the magnetic anisotropy changes from IMA to PMA, leading to a remarkable decrease of $H_{s}$.

For the ferromagnetic metal (FM)/Oxide heterostructures, the interfacial magnetic anisotropy plays a dominated role [16]. In theory, first-principles calculation has been used to study the FM/Oxide interface, showing that the interfacial magnetic anisotropy is strongly affected by the hybridization between FM-3d and O-2p orbits $[18,19]$. In addition, previous researches have reported that the orbital hybridization between FM and oxide is sensitive to the annealing process $[20,21]$. By annealing, the activated oxygen atoms could migrate to the interface, producing a bonding between FM atoms and oxygen atoms. It is necessary to point out that the degree of bonding is important to the orbital hybridization, where an optimized bonding is beneficial to PMA, whereas the excessive and insufficient bonding will lead to a degradation of PMA [22]. Here in our samples, the enthalpy of formation $\left(\Delta H_{f}\right)$ for $\mathrm{MgO}$ is $-601.6 \mathrm{~kJ} / \mathrm{mol}$, larger than that for $\mathrm{HfO}_{2}(-1144.7 \mathrm{~kJ} / \mathrm{mol})$. It means that the combination between $\mathrm{Hf}$ and $\mathrm{O}$ is more stable than that between $\mathrm{Mg}$ and $\mathrm{O}$. Therefore, during the deposition and annealing process, $\mathrm{MgO}$ is more likely to deviate the stoichiometric ratio and transfer oxygen atoms to the adjacent $\mathrm{CoFe}$ layer, leading to the final difference of the FM-O bonding degree for the two samples. According to our recent work, the oxygen migration direction during annealing process may be inverse at different FM/Oxide interfaces [23]. However, since the oxygen migration could also be affected by the film thickness and annealing temperature and so forth, the specific differences about oxygen migration in the two samples need further investigation.

In addition to $H_{s}$, AHE sensitivity is also related to $R_{\mathrm{AH}}$, whose value represents the magnitude of AHE. Previous work has reported that the annealing process will affect the intrinsic or extrinsic mechanisms, leading to a variation of AHE [24, 25]. To explain the change of $R_{\mathrm{AH}}$ in the MgOand $\mathrm{HfO}_{2}$-sample as shown in Figure 3, contributions to the AHE by different mechanisms were analyzed. In general, $\rho_{\mathrm{AH}}=a \rho_{x x}+b \rho_{x x}^{2}$, where $\rho_{\mathrm{AH}}$ is the saturated anomalous Hall resistivity, $\rho_{x x}$ is the longitudinal resistivity, $a$ represents the skew scattering contribution, and $b$ represents the side jump as well as the intrinsic contribution [26-30]. It is necessary to point out that the thickness change during annealing is eliminated; thus $\rho_{\mathrm{AH}}$ is equivalent to $R_{\mathrm{AH}}$. The coefficients $a$ and $b$ can be obtained by plotting $\rho_{\mathrm{AH}} / \rho_{x x}$ as a function of $\rho_{x x}$ and linear fitting to the experimental data. Figure 5(a) shows the linear fitting for $\mathrm{MgO}$-sample in the as-deposited and $240^{\circ} \mathrm{C}$ annealed states. The values of $a$ and $b$ are -0.029 and $2.12 \times 10^{-4} \mu \Omega^{-1} \mathrm{~cm}^{-1}$ in the as-deposited state, respectively. By annealing at $240^{\circ} \mathrm{C}$, the values of $a$ and $b$ change to 0.002 and $8.74 \times 10^{-5} \mu \Omega^{-1} \mathrm{~cm}^{-1}$, respectively. Although the sign of $a$ alters from negative to positive, both the values of $|a|$ and $|b|$ decrease by one order of magnitude, finally weakening the AHE. For the $\mathrm{HfO}_{2}$-sample, the values of $a$ and $b$ are -0.015 and $2.57 \times 10^{-4} \mu \Omega^{-1} \mathrm{~cm}^{-1}$ in the asdeposited state, respectively. By annealing at $240^{\circ} \mathrm{C}$, both the values of $|a|$ and $|b|$ increase by one order of magnitude, reaching -0.437 and $3.89 \times 10^{-3} \mu \Omega^{-1} \mathrm{~cm}^{-1}$, respectively. The competitive relation between $a$ and $b$ will affect not only the value but also the sign of $\rho_{\mathrm{AH}}$. Considering the large enhancement of $|b|$ as well as the same positive sign between $b$ and $\rho_{\mathrm{AH}}$, it suggests that the influence of $b$ on AHE is improved during annealing process for the $\mathrm{HfO}_{2}$-sample. Above analysis gives strong evidence that the variation trend of AHE is different during the annealing process in the $\mathrm{MgO}$ - and $\mathrm{HfO}_{2}$-sample. For the $\mathrm{MgO}$-sample, both the intrinsic and extrinsic contributions to AHE are weakened by annealing, resulting in the significant decrease of $R_{\mathrm{AH}}$ as shown in Figure 3(a). In contrast, the side jump and the intrinsic contributions are remarkably enhanced, leading to the final increase of $R_{\mathrm{AH}}$ as shown in Figure 3(b). 


\section{Conclusions}

In conclusion, the ultrasensitive AHE was demonstrated in $\mathrm{Ta} / \mathrm{CoFe} / O x i d e / \mathrm{Ta}$ multilayers. For sample $\mathrm{Ta} / \mathrm{CoFe} / \mathrm{MgO} /$ Ta, AHE sensitivity is as high as $18792 \Omega / \mathrm{T}$ in the as-deposited state, while the value decreases significantly as the annealing temperature increases. For sample $\mathrm{Ta} / \mathrm{CoFe} / \mathrm{HfO}_{2} / \mathrm{Ta}$, the value of sensitivity is small in the as-deposited state but increases to $14741 \Omega / \mathrm{T}$ by $240^{\circ} \mathrm{C}$ annealing. The opposite variation of AHE sensitivity in two samples originates from the different change of magnetic anisotropy and anomalous Hall resistance during the annealing process. This work gives new insights that both the choice of oxide material and the optimization of annealing treatment play an important role in the anomalous Hall sensitivity.

\section{Competing Interests}

The authors declare that there is no conflict of interests regarding the publication of this paper.

\section{Acknowledgments}

This work was supported by the National Basic Research Program of China (2015CB921502) and the Natural Science Foundation of China (51331002, 51371027, and 11504019).

\section{References}

[1] J. M. Daughton, "GMR and SDT sensor applications," IEEE Transactions on Magnetics, vol. 36, no. 5, pp. 2773-2778, 2000.

[2] C. P. O. Treutler, "Magnetic sensors for automotive applications," Sensors and Actuators, A: Physical, vol. 91, no. 1-2, pp. 2-6, 2001.

[3] S. G. Grancharov, H. Zeng, S. Sun et al., "Bio-functionalization of monodisperse magnetic nanoparticles and their use as biomolecular labels in a magnetic tunnel junction based sensor," The Journal of Physical Chemistry B, vol. 109, no. 26, pp. 1303013035, 2005.

[4] N. Nagaosa, J. Sinova, S. Onoda, A. H. MacDonald, and N. P. Ong, "Anomalous Hall effect," Reviews of Modern Physics, vol. 82, no. 2, pp. 1539-1592, 2010.

[5] Y. Tian, L. Ye, and X. Jin, "Proper scaling of the anomalous hall effect," Physical Review Letters, vol. 103, no. 8, Article ID 087206, 2009.

[6] J. Moritz, B. Rodmacq, S. Auffret, and B. Dieny, "Extraordinary Hall effect in thin magnetic films and its potential for sensors, memories and magnetic logic applications," Journal of Physics D: Applied Physics, vol. 41, no. 13, Article ID 135001, 2008.

[7] A. Gerber, “Towards Hall effect spintronics," Journal of Magnetism and Magnetic Materials, vol. 310, no. 2, pp. 2749-2751, 2007.

[8] Y. Zhu and J. W. Cai, "Ultrahigh sensitivity Hall effect in magnetic multilayers," Applied Physics Letters, vol. 90, no. 1, Article ID 012104, 2007.

[9] S. L. Zhang, J. Teng, J. Y. Zhang et al., "Large enhancement of the anomalous Hall effect in Co/Pt multilayers sandwiched by MgO layers," Applied Physics Letters, vol. 97, no. 22, Article ID 222504, 2010.
[10] J. Zhang, G. Yang, S. Wang et al., "Ultrahigh anomalous hall sensitivity in $\mathrm{Co} / \mathrm{Pt}$ multilayers by interfacial modification," Applied Physics Express, vol. 6, no. 10, Article ID 103007, 2013.

[11] Y. M. Lu, J. W. Cai, H. Y. Pan, and L. Sun, "Ultrasensitive anomalous Hall effect in $\mathrm{SiO}_{2} / \mathrm{Fe}-\mathrm{Pt} / \mathrm{SiO}_{2}$ sandwich structure films," Applied Physics Letters, vol. 100, no. 2, Article ID 022404, 2012.

[12] T. Zhu, P. Chen, Q. H. Zhang, R. C. Yu, and B. G. Liu, "Giant linear anomalous Hall effect in the perpendicular $\mathrm{CoFeB}$ thin films," Applied Physics Letters, vol. 104, no. 20, Article ID 202404, 2014.

[13] G. Kopnov and A. Gerber, "MegaOhm extraordinary Hall effect in oxidized CoFeB," Applied Physics Letters, vol. 109, no. 2, Article ID 022404, 2016.

[14] G. X. Miao and G. Xiao, "Giant Hall resistance in Pt-based ferromagnetic alloys," Applied Physics Letters, vol. 85, no. 1, pp. 73-75, 2004.

[15] T. Maeda, T. Kai, A. Kikitsu, T. Nagase, and J.-I. Akiyama, "Reduction of ordering temperature of an FePt-ordered alloy by addition of Cu," Applied Physics Letters, vol. 80, no. 12, pp. 2147$2149,2002$.

[16] S. Ikeda, K. Miura, H. Yamamoto et al., "A perpendicularanisotropy $\mathrm{CoFeB}-\mathrm{MgO}$ magnetic tunnel junction," Nature Materials, vol. 9, no. 9, pp. 721-724, 2010.

[17] E. Y. Vedmedenko, H. P. Oepen, and J. Kirschner, "Microstructure of the spin reorientation transition in second-order approximation of magnetic anisotropy," Physical Review B, vol. 66, no. 21, Article ID 214401, 2002.

[18] H. X. Yang, M. Chshiev, B. Dieny, J. H. Lee, A. Manchon, and K. H. Shin, "First-principles investigation of the very large perpendicular magnetic anisotropy at $\mathrm{Fe}-\mathrm{MgO}$ and $\mathrm{Co}-\mathrm{MgO}$ interfaces," Physical Review B, vol. 84, no. 5, Article ID 054401, 2011.

[19] K. H. Khoo, G. Wu, M. H. Jhon et al., "First-principles study of perpendicular magnetic anisotropy in $\mathrm{CoFe} / \mathrm{MgO}$ and $\mathrm{CoFe} /$ $\mathrm{Mg}_{3} \mathrm{~B}_{2} \mathrm{O}_{6}$ interfaces," Physical Review B, vol. 87, no. 17, Article ID 174403, 2013.

[20] X. Chen, C. Feng, Z. Long Wu et al., "Interfacial oxygen migration and its effect on the magnetic anisotropy in $\mathrm{Pt} / \mathrm{Co} / \mathrm{MgO} / \mathrm{Pt}$ films," Applied Physics Letters, vol. 104, no. 5, Article ID 052413, 2014.

[21] G. Yang, J.-Y. Zhang, S.-G. Wang et al., "Magnetization reorientation induced by interfacial structures in ultrathin disordered FePt film sandwiched by $\mathrm{SiO}_{2}$ layers," Applied Surface Science, vol. 353, pp. 489-493, 2015.

[22] A. Manchon, C. Ducruet, L. Lombard et al., "Analysis of oxygen induced anisotropy crossover in $\mathrm{Pt} / \mathrm{Co} / \mathrm{MOx}$ trilayers," Journal of Applied Physics, vol. 104, no. 4, Article ID 043914, 2008.

[23] G. Yang, J.-Y. Zhang, S.-L. Jiang et al., "Effect of oxygen migration on magnetic anisotropy and damping constant in perpendicular $\mathrm{Ta} / \mathrm{CoFeB} / \mathrm{Gd} / \mathrm{MgO} / \mathrm{Ta}$ multilayers," Applied Surface Science, 2016.

[24] S.-L. Jiang, X. Chen, X.-J. Li et al., "Anomalous Hall effect engineering via interface modification in Co/Pt multilayers," Applied Physics Letters, vol. 107, no. 11, Article ID 112404, 2015.

[25] S.-L. Jiang, G. Yang, J. Teng, Q.-X. Guo, L.-L. Li, and G.-H. Yu, "Interface-engineered spin-dependent transport in perpendicular Co/Pt multilayers," Applied Surface Science, vol. 387, pp. 375-378, 2016.

[26] M.-C. Chang and Q. Niu, "Berry phase, hyperorbits, and the Hofstadter spectrum: semiclassical dynamics in magnetic Bloch bands," Physical Review B - Condensed Matter and Materials Physics, vol. 53, no. 11, pp. 7010-7023, 1996. 
[27] D. Hou, Y. Li, D. Wei, D. Tian, L. Wu, and X. Jin, “The anomalous Hall effect in epitaxial face-centered-cubic cobalt films," Journal of Physics Condensed Matter, vol. 24, no. 48, Article ID 482001, 2012.

[28] J. Smit, “The spontaneous hall effect in ferromagnetics II," Physica, vol. 24, no. 1-5, pp. 39-51, 1958.

[29] L. Berger, "Side-jump mechanism for the hall effect of ferromagnets," Physical Review B, vol. 2, no. 11, pp. 4559-4566, 1970.

[30] A. Crépieux and P. Bruno, "Theory of the anomalous hall effect from the Kubo formula and the Dirac equation," Physical Review $B$, vol. 64, no. 1, Article ID 014416, 2001. 

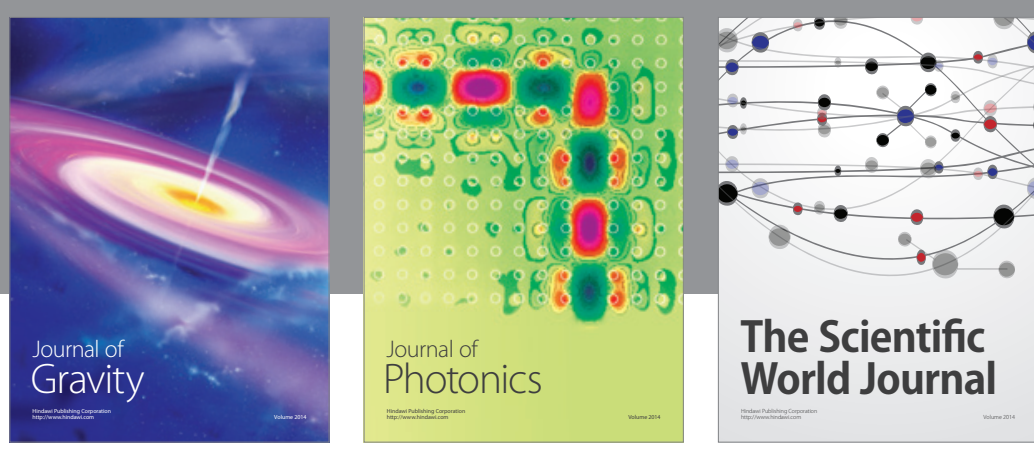

The Scientific World Journal
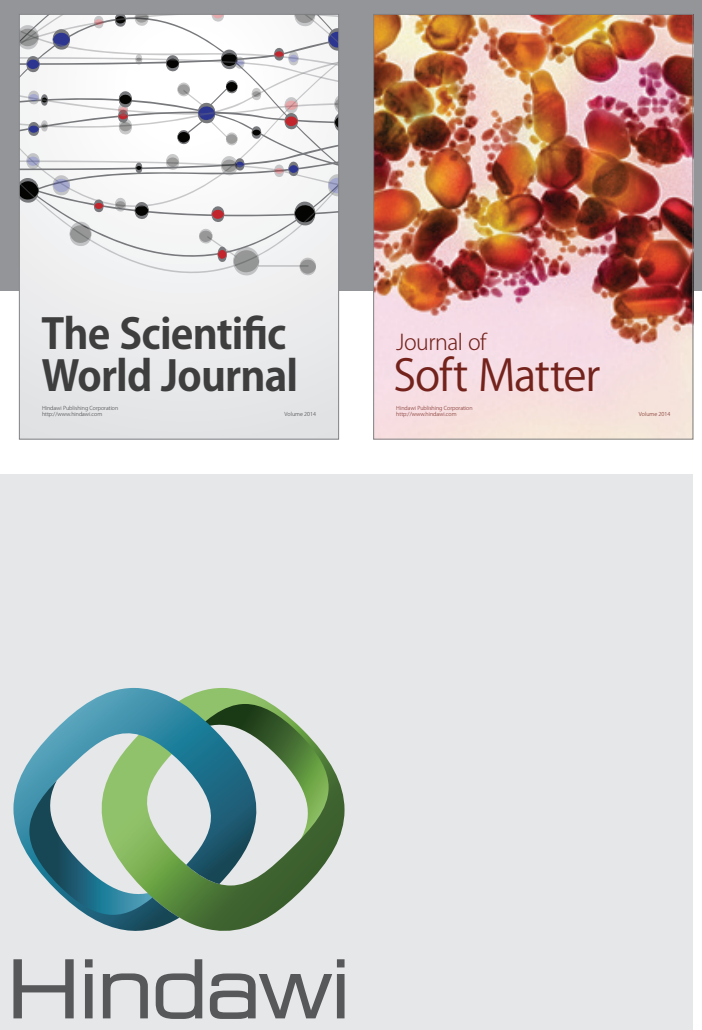

Submit your manuscripts at

http://www.hindawi.com

nternational Journal of

Statistical Mechanics
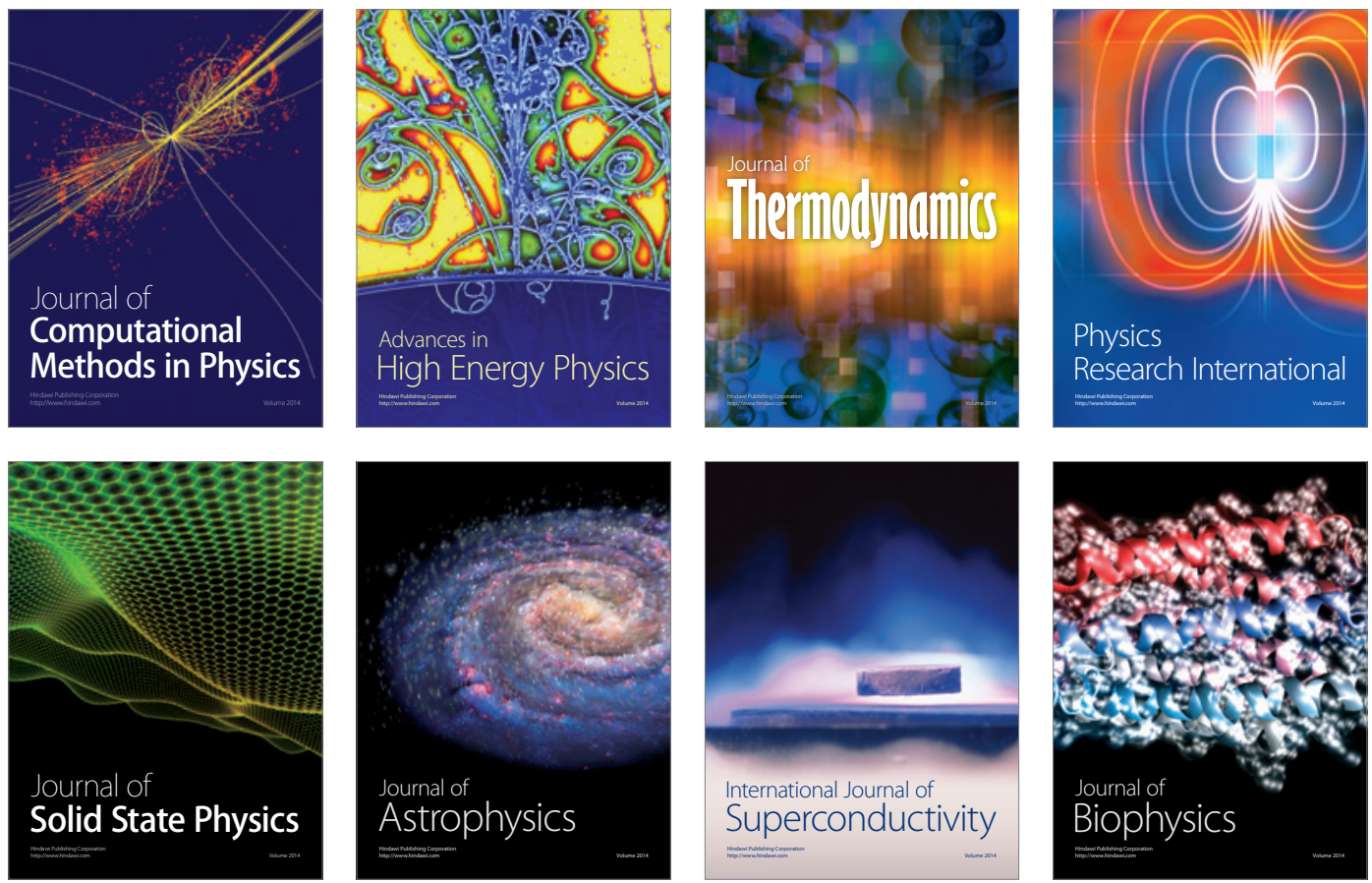
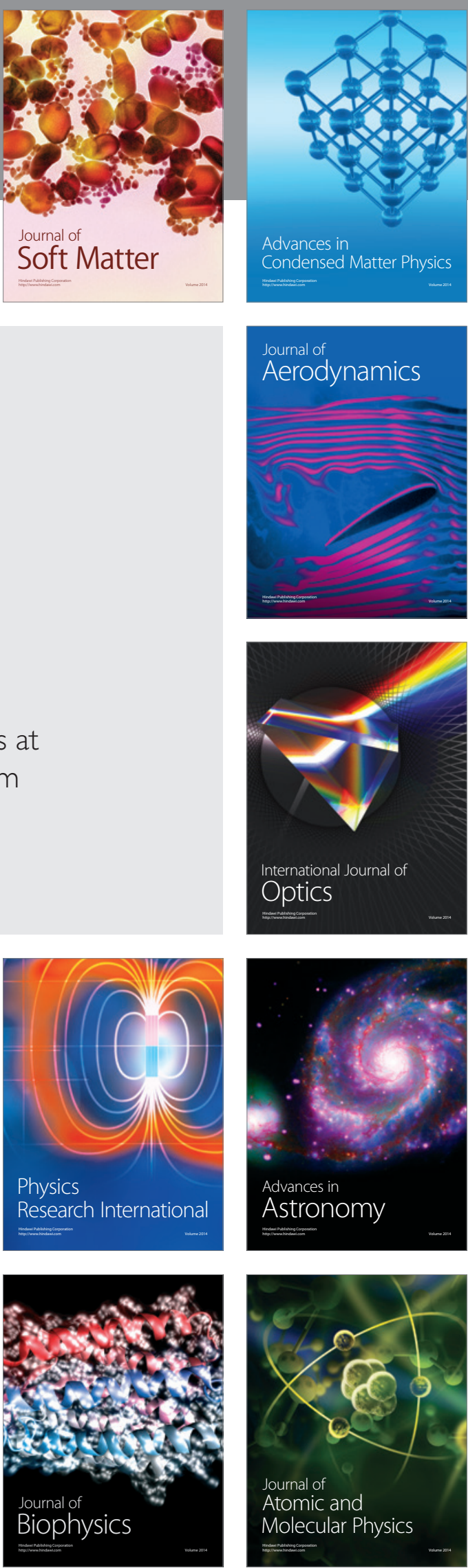